Research Paper

\title{
The Risk of Gynecologic and Urinary Tract Cancer with Pelvic Inflammatory Disease: A Population-Based Cohort Study
}

\author{
Hui-Wen Lin ${ }^{1,2^{*}}$, Po-Hui Wang $1,3,4^{*}$, Chung-Yuan Lee, ${ }^{1,5,6}$, Jing-Yang Huang7, Shun-Fa Yang 1,7, Yi-Hsuan \\ Hsiao $^{1,3,8}$ 凶 \\ 1. Institute of Medicine, Chung Shan Medical University, Taichung, Taiwan \\ 2. Department of Family Medicine, Taichung Hospital, Ministry of Health and Welfare, Taichung, Taiwan \\ 3. School of Medicine, Chung Shan Medical University, Taichung, Taiwan \\ 4. Department of Obstetrics and Gynecology, Chung Shan Medical University Hospital, Taichung, Taiwan \\ 5. Department of Obstetrics and Gynecology, Chiayi Chang Gung Memorial Hospital Chiayi, Taiwan \\ 6. Department of Nursing, Chang Gung University of Science and Technology, Chiayi Campus, Chiayi, Taiwan \\ 7. Department of Medical Research, Chung Shan Medical University Hospital, Taichung, Taiwan \\ 8. Department of Obstetrics and Gynecology, Changhua Christian Hospital, Changhua, Taiwan \\ *These authors contributed equally to the work. \\ $\square$ Corresponding author: Yi-Hsuan Hsiao, MD., Ph.D., Department of Obstetrics and Gynecology, Changhua Christian Hospital, Changhua, Taiwan; E-mail: \\ 54315@cch.org.tw \\ (c) Ivyspring International Publisher. This is an open access article distributed under the terms of the Creative Commons Attribution (CC BY-NC) license \\ (https://creativecommons.org/licenses/by-nc/4.0/). See http://ivyspring.com/terms for full terms and conditions.
}

Received: 2018.08.16; Accepted: 2018.10.05; Published: 2019.01.01

\begin{abstract}
Purpose: The aim of the study was to determine the association between pelvic inflammatory disease (PID) and breast, colorectal, gynecologic and urinary tract cancer.

Methods: The source of data was a longitudinal dataset compiled by the Longitudinal Health Insurance Database 2000 (LHID2000) which was collected by the National Health Insurance program. Cases of PID, at least two outpatient visits and one admission, were diagnosed from 2000 to 2013. The data for controls, age matched women who were not diagnosed with PID from 2000 to 2013 , were also obtained.

Results: A total of 47,333 PID cases and 189,332 for control group were included in the study. The ectopic pregnancy incidence rate (per 10000 person months) was 1.912 and 0.595 in the PID and control group, respectively; the rate ratio was 3.211 (confidence interval, $\mathrm{Cl}=2.931-3.519$ ). There were significantly different in gynecologic cancers and urinary tract cancers between the PID and control group; the rate ratios (95\% C.I.) were 1.903 (1.672-2.166) and 1.566 (1.211-2.025), respectively.

Conclusion: Our study found that PID was associated with increased risks of gynecologic and urinary tract cancer but not colorectal or breast cancer. The information may lead to a strategy for cancer prevention and a reasonable healthcare usage through understanding PID epidemiology and controlling the inflammatory responses.
\end{abstract}

Key words: breast, colorectal, gynecologic, urinary tract cancer, pelvic inflammatory disease

\section{Introduction}

Pelvic inflammatory disease (PID) is an infection-induced inflammation disease of the female upper genital tract. It involves any combination of the uterine endometrium, fallopian tubes, ovaries and pelvic peritoneum [1]. Long-term complications of PID and adverse sequelae after PID are concerned in clinical care for its inflammation resulting in tubal scarring and damage which lead to reproductive 
disability, such as ectopic pregnancy and infertility [2].

Cancer linked to inflammation was perceived since the 19th century [3]. Inflammation in selected organ may increase the risk of cancer [3]. Cancerrelated inflammation links to genetic instability by inflammatory mediators [3]. Molecular pathways, which link inflammation and cancer, have been reported [4-6]. Tumor microenvironment is an indispensable participant in the neoplastic process, proliferation and migration; some of the signaling molecules of the innate immune system are co-opted by tumor cells. Therefore, inflammation is a crucial component of tumor progression [4]. Inflammatory cells and cytokines in tumors could contribute to tumor growth, progression, and immunosuppression [5]. The mediators and cellular effectors of inflammation play important roles for local environment of tumors [6]. Inflammation has been established as an enabling characteristic of cancer and as a hallmark of cancer by molecular biology evidence [7].

Epidemiology researches for the association of PID with cancers have been investigated [8]; researches involving PID in association with a certain type of gynecologic cancers have been proposed. However, the results are not all consistent [8-16]. More investigations are required to clarify the risk of diseases for PID patients [13]. Understanding PID epidemiology leads to reasonable healthcare usage.

The aim of the present study was to investigate

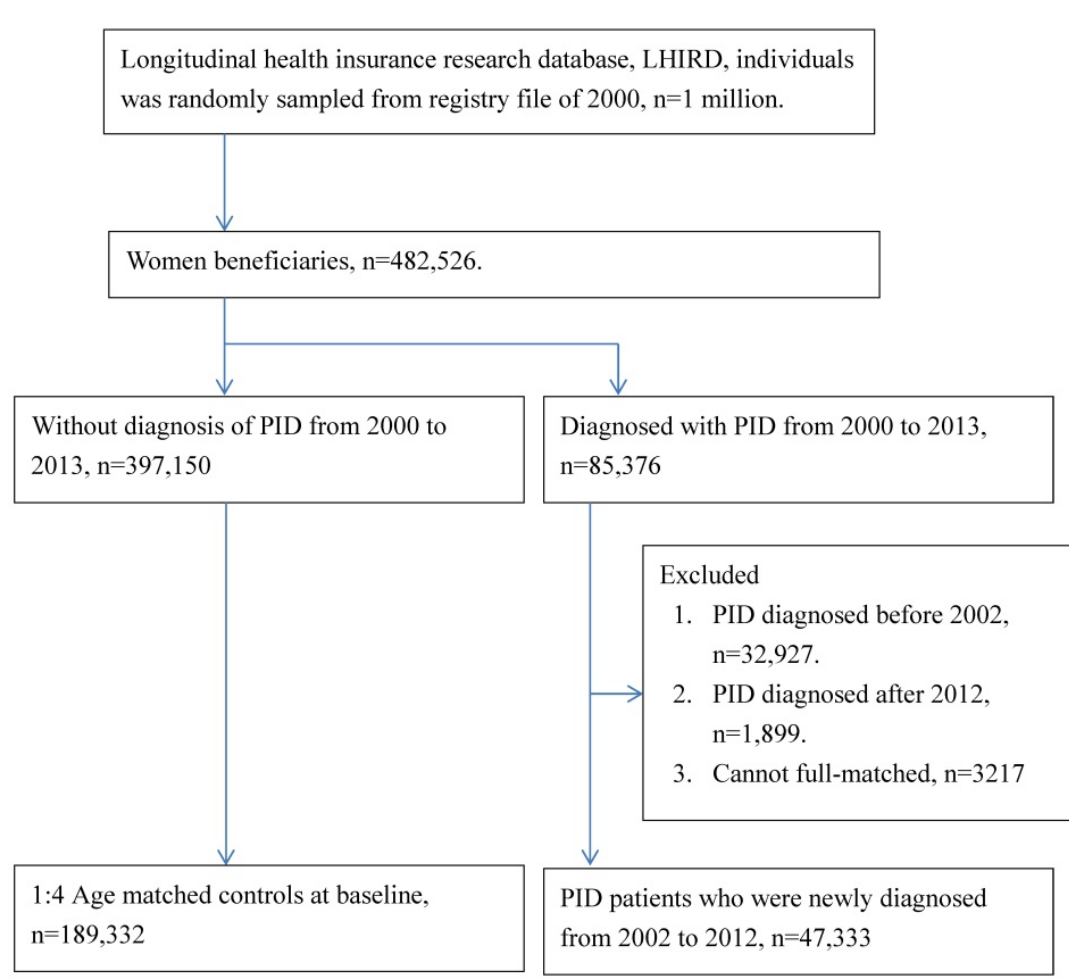

Figure 1. Flow chart of this study. PID, pelvic inflammatory disease the risks of breast cancer, colorectal cancer, gynecologic cancer and urinary tract cancer in the female patients with PID by a population-based cohort study.

\section{Materials and Methods}

\section{Data source}

The source of data was a longitudinal dataset compiled by the Longitudinal Health Insurance Database 2000 (LHID2000). The LHID2000 contains registration and claim data of 1,000,000 individuals, randomly sampled from registry file of 2000 , collected by the National Health Insurance program. There were no significant differences between the patients in the LHID2000 and the original National Health Insurance Research Database (NHIRD) in general distribution. (https://nhird.nhri.org.tw/en/Data_ Subsets.html). The NHIRD contains comprehensive information regarding clinical detailed and diagnostic codes based on the International Statistical Classification of Diseases and Related Health Problems codes (ninth revision, clinical modification), namely ICD-9-CM codes.

\section{Study population}

This is a retrospective cohort study. Flow chart of this study was shown in Figure 1. Cases of PID were diagnosed, individuals with ICD-9-CM code 614,615 (at least two outpatient visits and one admission), from 2000 to 2013 in the national health insurance dataset according to the characteristic criteria of national guidelines for PID. Those PID cases diagnosed before 2002, after 2012 or not been full-matched were excluded. The data for controls, age matched women who were not diagnosed with PID from 2000 to 2013, were also obtained.

The main outcomes of our study included ectopic pregnancy (ICD-9: 633), colorectal cancer (ICD-9: 154), breast cancer (ICD-9: 174), gynecologic cancer (ICD-9: 179-184) and urinary tract cancer (ICD-9:188-189). The considered variables of our study included age at baseline (matching variable), urbanization (urban, suburban, rural), income (non-low, low income), outpatient visit within 2 years before baseline $(0,1-13,14-27,28-55,>=56)$, length of hospital stay within 2 years before baseline $(0,1-6,7-13,>=14)$. The co-morbidities were also concerned in this study, including asthma (ICD-9: 
493), atopic dermatitis (ICD-9: 691.0), chronic liver disease (ICD-9: 571), peptic ulcer (ICD-9: 531-534), zoster (ICD-9: 053, 054), hepatitis B virus infection (ICD-9: 070.2, 070.3, V02.61), hepatitis C virus infection (ICD-9: 070.44, 070.51, 070.54, 070.7, V02.62), gout (ICD-9: 274), chronic lung diseases (ICD-9: 490-492 495 496, 500-508), cerebrovascular diseases (ICD-9: 430-438), ischemic heart diseases (ICD-9: 410-414), congestive heart failure (ICD-9: 428, 402.01, 402.11, 402.91, 404.01, 404.03, 404.11, 404.13, 404.91, 404.93, 429.4, 398.91), renal failure (ICD-9: 40301, 40401, 40403, 40411, 40413, 40491, 40493, 584, 585, 586), urinary tract infection (ICD-9: 590, 595, 597, 5990).

\section{Follow up}

For each specific main event, the prevalent cases were excluded from the baseline. All cases, including the PID and control, were followed up until event occurrence, death, or end of study (Dec, 2013).

\section{Statistical analysis}

The two-tailed student $\mathrm{t}$ test and chi-square test were used for comparing the difference of continue and category variables between PID and control group. The time to event analysis was performed to estimate the incidence risk of main events between groups.

The hazard ratios (HRs) of main events with PID exposure estimated by Cox proportional hazard model in different statistical modeling, including univariate, multivariate modeling and inverse propensity score weighting. The inverse propensity score weighting Cox proportional hazard models was conducted to explore the hazard ratios, while the confounding bias may be exist in our study. KM plotter evaluation of main outcomes (ectopic pregnancy, colorectal cancer, breast cancer, gynecologic cancer, and urinary tract cancer) between the PID and control were also conducted.

\section{Results}

A total of 47,333 PID cases and 189,332 for control group were included in this study (Figure 1). The characteristics between the PID and control group were shown in Table 1 . The significant variables existed between the PID and control group, including urbanization, low income, outpatient visits, length of hospital stay, comorbidities, old events (ectopic pregnancy, colorectal cancer, breast cancer, gynecologic cancers and urinary tract cancers) before baseline.

The incidence rates and rate ratios of main outcomes for the PID and control groups were shown in Table 2. The ectopic pregnancy incidence rate (per 10000 person months) was 1.912 and 0.595 in the PID and control group, respectively; the rate ratio was
3.211 (confidence interval, $\mathrm{CI}=2.931-3.519$ ). There were significantly different in gynecologic cancer and urinary tract cancer between the PID and control group (Table 2); the rate ratios (95\% C.I.) were 1.903 (1.672-2.166) and 1.566 (1.211-2.025), respectively. However, there were no significant differences in colorectal cancer and breast cancer between the PID and control group; the rate ratios (95\% C.I.) were 1.281 (0.989-1.659) and 1.030 (0.923-1.150), respectively.

Table 1. baseline characteristics of PID and control groups.

\begin{tabular}{|c|c|c|c|}
\hline & \multicolumn{2}{|l|}{ Group } & \multirow[b]{2}{*}{ p value } \\
\hline & $\begin{array}{l}\text { Control } \\
\mathrm{N}=189,332\end{array}$ & $\begin{array}{l}\text { PID } \\
\mathrm{N}=47,333\end{array}$ & \\
\hline Age at baseline & & & 1.0000 \\
\hline$<15$ & $936(0.49 \%)$ & $234(0.49 \%)$ & \\
\hline $15-24$ & $41004(21.66 \%)$ & $10251(21.66 \%)$ & \\
\hline $25-34$ & $51968(27.45 \%)$ & $12992(27.45 \%)$ & \\
\hline $35-44$ & $48320(25.52 \%)$ & $12080(25.52 \%)$ & \\
\hline $45-54$ & $31448(16.61 \%)$ & $7862(16.61 \%)$ & \\
\hline $55-64$ & $9648(5.1 \%)$ & $2412(5.1 \%)$ & \\
\hline$>=65$ & $6008(3.17 \%)$ & $1502(3.17 \%)$ & \\
\hline Urbanization & & & $<0.0001$ \\
\hline Urban & $120550(63.67 \%)$ & $28975(61.22 \%)$ & \\
\hline Sub-urban & $53242(28.12 \%)$ & $14062(29.71 \%)$ & \\
\hline Rural & $15540(8.21 \%)$ & $4296(9.08 \%)$ & \\
\hline Low income & $1098(0.58 \%)$ & $353(0.75 \%)$ & $<0.0001$ \\
\hline Outpatient visits & & & $<0.0001$ \\
\hline 0 & $11526(6.09 \%)$ & $184(0.39 \%)$ & \\
\hline $1-13$ & $58988(31.16 \%)$ & $7229(15.27 \%)$ & \\
\hline $14-27$ & $55537(29.33 \%)$ & $13854(29.27 \%)$ & \\
\hline $28-55$ & $46680(24.66 \%)$ & $17638(37.26 \%)$ & \\
\hline$>=56$ & $16601(8.77 \%)$ & $8428(17.81 \%)$ & \\
\hline Length of hospital stay & & & $<0.0001$ \\
\hline 0 & $167806(88.63 \%)$ & $38465(81.26 \%)$ & \\
\hline $1-6$ & $15674(8.28 \%)$ & $6667(14.09 \%)$ & \\
\hline $7-13$ & $3426(1.81 \%)$ & $1437(3.04 \%)$ & \\
\hline$>=14$ & $2426(1.28 \%)$ & $764(1.61 \%)$ & \\
\hline \multicolumn{4}{|l|}{ Old event before baseline } \\
\hline Ectopic pregnancy & $758(0.40 \%)$ & $656(1.39 \%)$ & $<0.0001$ \\
\hline Colorectal cancer & $115(0.06 \%)$ & $36(0.08 \%)$ & 0.2379 \\
\hline Breast cancer & $809(0.43 \%)$ & $214(0.45 \%)$ & 0.4615 \\
\hline Gynecologic cancer & $532(0.28 \%)$ & $317(0.67 \%)$ & $<0.0001$ \\
\hline Urinary tract cancer & $100(0.05 \%)$ & $47(0.10 \%)$ & 0.0003 \\
\hline \multicolumn{4}{|l|}{ Co-morbidities } \\
\hline Asthma & $5925(3.13 \%)$ & $2003(4.23 \%)$ & $<0.0001$ \\
\hline Atopic dermatitis & $269(0.14 \%)$ & $121(0.26 \%)$ & $<0.0001$ \\
\hline Chronic liver disease & $10093(5.33 \%)$ & $3503(7.40 \%)$ & $<0.0001$ \\
\hline Peptic ulcer & $16336(8.63 \%)$ & $6599(13.94 \%)$ & $<0.0001$ \\
\hline Zoster & $5487(2.90 \%)$ & $1816(3.84 \%)$ & $<0.0001$ \\
\hline Hepatitis B virus infection & $2559(1.35 \%)$ & $854(1.80 \%)$ & $<0.0001$ \\
\hline Hepatitis C virus infection & $701(0.37 \%)$ & $241(0.51 \%)$ & $<0.0001$ \\
\hline Gout & $3228(1.70 \%)$ & $1109(2.34 \%)$ & $<0.0001$ \\
\hline Chronic lung diseases & $9921(5.24 \%)$ & $3402(7.19 \%)$ & $<0.0001$ \\
\hline Cerebrovascular diseases & $2431(1.28 \%)$ & $757(1.60 \%)$ & $<0.0001$ \\
\hline Ischemic heart diseases & $4649(2.46 \%)$ & $1764(3.73 \%)$ & $<0.0001$ \\
\hline Congestive heart failure & $1323(0.70 \%)$ & $469(0.99 \%)$ & $<0.0001$ \\
\hline Renal failure & $909(0.48 \%)$ & $267(0.56 \%)$ & 0.0201 \\
\hline Urinary tract infection & $23788(12.56 \%)$ & $12860(27.17 \%)$ & $<0.0001$ \\
\hline
\end{tabular}

PID, pelvic inflammatory disease

The hazard ratios (HRs) of main events with PID exposure were shown in Table 3. There were no significant difference between the PID and control group in the hazard ratio of breast and colorectal 
cancer by univariate, multivariate modeling and inverse propensity score weighting analyses. Hazard ratio of gynecologic cancer in PID group was significantly different from the control group by using varied modeling analyses (Table 3 ). In analyzing the hazard ratio of urinary tract cancer, there was no statistically significance between the PID and control group by multivariate modeling analysis; there were statistically significance between the PID and control group by univariate modeling and inverse propensity score weighting analyses. By using inverse propensity score weighting model, the main outcomes, including ectopic pregnancy, gynecologic cancer and urinary tract cancer, showed significant differences between the PID and control group.

KM plotter evaluations of main outcomes (ectopic pregnancy, colorectal cancer, breast cancer, gynecologic cancer, urinary tract cancer) between the PID and control group were shown in Figure 2.

\section{Discussion}

Our study found that PID was associated with increasing risks of ectopic pregnancy, gynecologic cancer and urinary tract cancer but not colorectal cancer or breast cancer. Most cases of gynecologic cancer were diagnosed within one year after episode of PID.

The result involving the association of PID with ectopic pregnancy of our study is consistent with previous studies $[17,18]$. Tubal damage caused by PID increases the risk of ectopic pregnancy and infertility. The association of tubal factor infertility after PID with number and severity of PID episodes was reported by a cohort study using diagnostic laparoscopy (index laparoscopy) to verify disease for clinical suspicious of acute PID patients [17]. The authors also demonstrated that the incidences of ectopic pregnancy in the first pregnancy patients after laparoscopically-confirmed PID and those without PID at laparoscopy were $9.1 \%$ and $1.4 \%$, respectively [17]. The increased expression of uteroglobin in damaged fallopian tubes by PID was proposed by Quintar et al [19]. The authors claimed that situation may play an important role in ectopic pregnancy.

The association between PID and colorectal cancer was shown by a matched cohort study through 5 -year follow-up period by using the data from the Longitudinal Health Insurance Database 2005 (LHID2005) in Taiwan [20]. The adjusted hazard ratio of colorectal cancer risk for women with PID compared to the comparison cohort was 1.71 (95\% CI 1.10-2.65) [20]. The researchers constructed the PID patient group by at least two consensus diagnosis of PID and excluded women younger 13 years or older than 45 years of age. Some important information of the study subjects was not contained in the Database, and this led to a limitation of uniform diagnosis of PID [21, 22]. Re-analyzing biological gradient was performed by the researchers and the colorectal cancer probabilities were related to frequencies of PID visit [22]. The odds ratio of colorectal cancer during visits $1-12$ and $>12$ visits for PID patients compared with the control group were $1.72(p=0.019)$ and 2.84 $(p=0.043)$, respectively [22]. In our study, we defined the PID patients with at least two outpatient visits and one admission. Rate ratio of colorectal cancer in PID and control groups were 1.281 (Table 2). Adjusted hazard ratio of colorectal cancer with PID exposure, estimated by Cox proportional hazard model in inverse propensity score weighting model, revealed 1.185. There was no significant association between PID and colorectal cancer also shown by KM plotter evaluation (Figure 2).

The association between PID and ovarian cancer was firstly reported by Risch and Howe. A case-control study, including 450 cases of epithelial ovarian cancer and 565 population controls during 1989-1992, was conducted [23]. The authors showed that PID increased the risk of developing epithelial ovarian cancer and inflammatory changes of the ovarian epithelium played a crucial role for the association [23]. However, Parazzini et al. analyzed the association between history of PID and the risk of subsequent epithelial ovarian cancer by conducting a case-control study during 1983-1991 in Italy [24]. The cases studied were 971 females with epithelial ovarian cancer and control subjects were 2758 women. The authors demonstrated absence of association between history of PID and risk of ovarian cancer [24].

Table 2. Incidence rates of main outcomes in PID and control groups.

\begin{tabular}{|c|c|c|c|c|c|c|c|c|c|}
\hline & \multicolumn{4}{|c|}{ Control $\mathrm{N}=189,332$} & \multicolumn{4}{|c|}{ PID N=47,333 } & \multirow[b]{2}{*}{$\begin{array}{l}\text { Rate ratio (95\% } \\
\text { C.I.) }\end{array}$} \\
\hline & $\begin{array}{l}\text { At risk } n \text { at } \\
\text { baseline }\end{array}$ & $\begin{array}{l}\text { Follow up } \\
\text { person months }\end{array}$ & Event & $\begin{array}{l}\text { Incidence } \\
\text { rate* }^{*}\end{array}$ & $\begin{array}{l}\text { At risk } n \text { at } \\
\text { baseline }\end{array}$ & $\begin{array}{l}\text { Follow up } \\
\text { person months }\end{array}$ & Event & $\begin{array}{l}\text { Incidence } \\
\text { rate* }\end{array}$ & \\
\hline \multicolumn{10}{|l|}{ Main outcome } \\
\hline Ectopic pregnancy & 188409 & 17366854 & 1034 & 0.595 & 46739 & 4314786 & 825 & 1.912 & $3.211(2.931-3.519)$ \\
\hline Colorectal cancer & 189202 & 17483616 & 235 & 0.134 & 47290 & 4412875 & 76 & 0.172 & $1.281(0.989-1.659)$ \\
\hline Breast cancer & 188453 & 17366531 & 1539 & 0.886 & 47110 & 4380708 & 400 & 0.913 & $1.030(0.923-1.150)$ \\
\hline Gynecologic cancer & 188712 & 17419788 & 709 & 0.407 & 47013 & 4377291 & 339 & 0.774 & $1.903(1.672-2.166)$ \\
\hline Urinary tract cancer & 189204 & 17482875 & 205 & 0.117 & 47295 & 4411713 & 81 & 0.184 & $1.566(1.211-2.025)$ \\
\hline
\end{tabular}


Table 3. Hazard ratio of main events with PID exposure, estimated by Cox proportional hazard model in different statistical modeling.

\begin{tabular}{|c|c|c|c|c|c|c|}
\hline & \multicolumn{6}{|c|}{ Hazard ratio (95 \% C.I.) for main events with PID exposure in different model } \\
\hline & \multicolumn{2}{|c|}{ Univariate modeling } & \multicolumn{2}{|c|}{ Multivariate modeling $\dagger$} & \multicolumn{2}{|c|}{ Inverse propensity score weighting } \\
\hline & HR (95 \% C.I.) & p value & aHR (95 \% C.I.) & p value & HR (95 \% C.I.) & p value \\
\hline \multicolumn{7}{|l|}{ Main outcome } \\
\hline Ectopic pregnancy & $3.210(2.929-3.518)$ & $<0.0001$ & $2.976(2.702-3.278)$ & $<0.0001$ & $3.208(2.930-3.512)$ & $<0.0001$ \\
\hline Colorectal cancer & $1.281(0.989-1.659)$ & 0.0606 & $1.237(0.946-1.617)$ & 0.1205 & $1.185(0.908-1.547)$ & 0.2121 \\
\hline Breast cancer & $1.030(0.922-1.149)$ & 0.6042 & $1.020(0.910-1.142)$ & 0.7343 & $0.985(0.881-1.101)$ & 0.7871 \\
\hline Gynecologic cancer & $1.904(1.673-2.167)$ & $<0.0001$ & $2.018(1.763-2.310)$ & $<0.0001$ & $2.107(1.859-2.389)$ & $<0.0001$ \\
\hline Urinary tract cancer & $1.567(1.212-2.027)$ & 0.0006 & $1.233(0.945-1.61)$ & 0.1233 & $1.322(1.011-1.728)$ & 0.0412 \\
\hline
\end{tabular}

† The covariates included age group, urbanization, income, medical utilization (like outpatient visit or hospitalization within 2 years before baseline), co-morbidities at baseline. aHR: Adjusted hazard ratios; HR: hazard ratios; PID, pelvic inflammatory disease; 95\% C.I. , 95\% confidence interval

(A) ectopic pregnancy

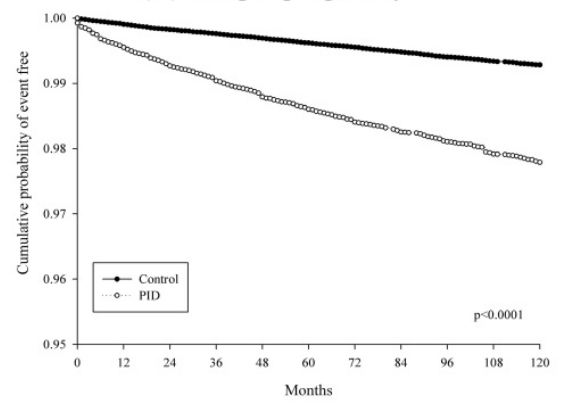

(D) gynecologic cancer

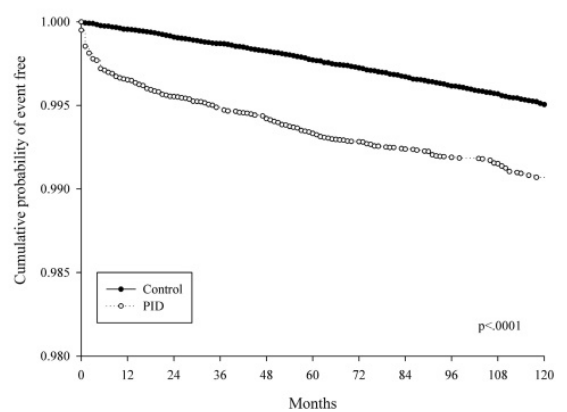

(B) colorectal cancer

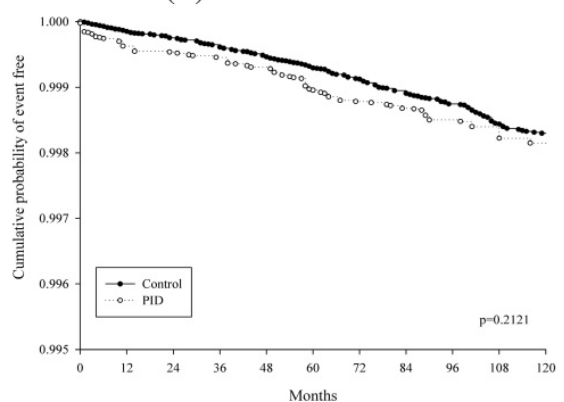

(C) breast cancer

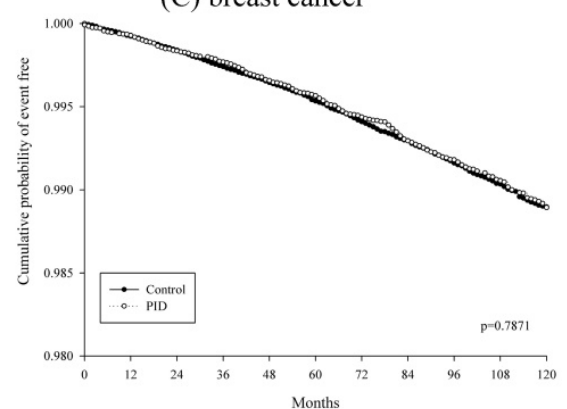

(E) urinary tract cancer

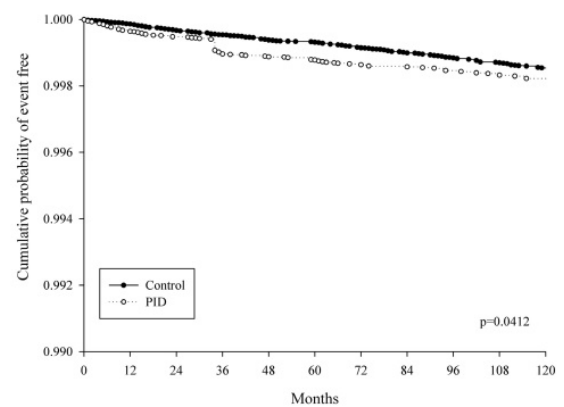

Figure 2. KM plotter evaluations of main outcomes (A) ectopic pregnancy, (B) colorectal cancer, (C) breast cancer, (D) gynecologic cancer and (E) urinary tract cancer between the PID and control.

Lin et al. showed an increased incidence of ovarian cancer in those patients with a history of PID (HR 1.92, 95\% CI 1.27-2.92) during a follow-up period of three years [15]. The study cohort included ICD-9-CM code 614, 615 PID patients; nevertheless, ICD-9-CM code 616 refers to the inflammatory disease of lower genital tract, i.e. cervix, vagina, and vulva. Wrong classification of study population could exist in this survey [25]. Previous study also shown that the prevalence of PID with endometriosis is higher than the general population [26]. Some studies demonstrated the risks of breast, ovarian and uterine cancers following pelvic inflammatory disease [14, 27]. The authors selected the patients by at least two consensuses PID for the study group. The adjusted hazard ratio of ovarian, breast or uterine cancer in PID group were: HR 1.326, HR: 1.39, and HR: 1.439 respectively in comparison with controls. The researchers concluded that there were no increased risks for ovarian, breast or uterine cancer among women with PID compared to the control group [14].

The role of inflammatory change in ovarian cancer has been surveyed on the point of molecular biology [9]. The authors focused on activated B cells to explain pathophysiology of inflammation and cancer. Chronic inflammation is a crucial role in the pathogenesis of ovarian cancer. Immune cells, fibroblasts, the extracellular matrix and other various components all constitute the tumor stroma [28]. Cancer-associated fibroblasts (CAFs) are one of the components of the tumor microenvironment [28]. The biochemical interactions of cancers cells with their microenvironment may play a critical role in spreading aggressive cancer cells from the primary tumor [29]. Inflammatory cells influence tumor microenvironment which is essential for cancer progression, growth, invasion and 
metastasis [28] Cancer-associated fibroblasts play a key role in tumor initiation, progression, and metastasis by modifying the components of the inflammatory microenvironment and mediating tumor-promoting inflammation [7].

The cause of ovarian cancer is still unclear [30]. The cellular microenvironment on the development of the ovarian cancer has been emphasized [31]. The oncogenic RAS-transformed ovarian epithelial cells generate pro-inflammatory secretome which diffuse into the stromal and cause fibroblasts senescence. Senescence of ovarian stromal fibroblasts create a secondary pro-inflammatory phenotype and convert the ovarian epithelial microenvironment into a field filled with inflammatory mediators that advance tumor progressing. The inflammatory network contribute the communication between the ovarian tumor epithelium and underlying stromal [31].

The role of inflammation in cancer progression on the ovarian cancer was emphasized [9]. The potential role of infection agents as well as PID in ovarian carcinogenesis has been examined by seven epidemiological studies [32]. Potential carcinogenic bacteria were detected; however, inconsistent results of the association between bacterial infection and ovarian cancer were found. Further researches were warranted [32].

Patients suffering from PID have a higher risk of cervical cancer was reported [33]. PID may be a risk factor for cervical cancer by examining the relationship PID and human papillomavirus (HPV). The potential of chronic PID as a risk factor to cervical dysplasia was examined the cervical smear pattern in patients with chronic PID [34]. The results showed there were higher cases of dysplasia in the chronic PID group than in the control. The authors claimed that cervical cancer screening programs should be intensified in chronic PID patients [34].

Our study provides epidemiologic evidence that PID associates with increased risks of gynecologic and urinary tract cancer but not colorectal or breast cancer. The useful information may lead to a strategy for cancer prevention and a reasonable healthcare usage through understanding PID epidemiology and controlling the inflammatory responses.

The major limitations of this study are our inability to include taking medicine information which may reduce the risk of developing cancers, including oral contraceptives and raloxifene. Use of oral contraceptives reduces the incidence rate of endometrial, ovarian, and colorectal cancer [35-37]. Raloxifene treatment reduces the incidence of breast cancer in postmenopausal women with osteoporosis and the outcome relevant to raloxifene continues beyond 4 years of treatment [38]. Another limitation of our study was unable to evaluate the effect of different determination of PID. The clinical presentations of PID range from subclinical infection to severe situation which requires hospital admission for procedural intervention or intravenous antibiotics [39]. In this study, the cases with at least two outpatient visits and one admission were evaluated. Those cases with subclinical infection could be misclassified.

In conclusion, this study revealed PID associated with increased risks of gynecologic cancer and urinary tract cancer but not colorectal cancer or breast cancer. Most cases of gynecologic cancer were diagnosed within one year after episode of PID. This could be due to the procedure of PID diagnosis involving the notification of gynecologic cancer. Further investigations involving large studies using time interval analyses and separate analyses for different types of cancers are required to verify the association of PID with gynecologic cancer.

\section{Acknowledgements}

This study is based in part on data from the National Health Insurance Research Database provided by the Bureau of National Health Insurance, Department of Health and managed by National Health Research Institutes (https://nhird.nhri.org. tw/en/Data_Subsets.html). The interpretation and conclusions contained herein do not represent those of National Health Insurance Administration, Department of Health or National Health Research Institutes." This work was supported by grant 105-CCH-IRP-045 from the Changhua Christian Hospital Research Foundation.

\section{Competing Interests}

The authors have declared that no competing interest exists.

\section{References}

[1] Mitchell C, Prabhu M. Pelvic inflammatory disease: current concepts in pathogenesis, diagnosis and treatment. Infect Dis Clin North Am 2013; 27: 793-809.

[2] Brunham RC, Gottlieb SL, Paavonen J. Pelvic inflammatory disease. N Engl J Med 2015; 372: 2039-2048.

[3] Colotta F, Allavena P, Sica A, Garlanda C, Mantovani A. Cancer-related inflammation, the seventh hallmark of cancer: links to genetic instability. Carcinogenesis 2009; 30: 1073-1081.

[4] Coussens LM, Werb Z. Inflammation and cancer. Nature 2002; 420: 860-867.

[5] Balkwill F, Mantovani A. Inflammation and cancer: back to Virchow? Lancet 2001; 357: 539-545

[6] Mantovani A, Allavena P, Sica A, Balkwill F. Cancer-related inflammation. Nature 2008; 454: 436-444.

[7] Servais C, Erez N. From sentinel cells to inflammatory culprits: cancer-associated fibroblasts in tumour-related inflammation. J Pathol 2013; 229: 198-207.

[8] Rasmussen $C B$, Jensen A, Albieri V Andersen KK, Kjaer SK. Is Pelvic Inflammatory Disease a Risk Factor for Ovarian Cancer? Cancer Epidemiol Biomarkers Prev 2017; 26: 104-109.

[9] Kisielewski R, Tolwinska A, Mazurek A, Laudanski P. Inflammation and ovarian cancer--current views. Ginekol Pol 2013; 84: 293-297. 
[10] Zhou Z, Zeng F, Yuan J, Tang J, Colditz GA, Tworoger SS, et al. Pelvic inflammatory disease and the risk of ovarian cancer: a meta-analysis. Cancer Causes Control 2017; 28: 415-428.

[11] Rasmussen CB, Faber MT, Jensen A, Høgdall E, Høgdall C, Blaakær J, et al. Pelvic inflammatory disease and risk of invasive ovarian cancer and ovarian borderline tumors. Cancer Causes \& Control 2013; 24: 1459-1464.

[12] Rasmussen CB, Jensen A, Albieri V, Andersen KK, Kjaer SK. Increased risk of borderline ovarian tumors in women with a history of pelvic inflammatory disease: A nationwide population-based cohort study. Gynecol Oncol 2016; 143: 346-351.

[13] Rasmussen CB, Kjaer SK, Albieri V, Bandera EV, Doherty JA, Hogdall E, et al. Pelvic Inflammatory Disease and the Risk of Ovarian Cancer and Borderline Ovarian Tumors: A Pooled Analysis of 13 Case-Control Studies. Am J Epidemiol 2017; 185: 8-20.

[14] Shen CC, Hu LY, Yang AC, Chiang YY, Hung JH, Tsai SJ. Risk of uterine, ovarian and breast cancer following pelvic inflammatory disease: a nationwide population-based retrospective cohort study. BMC Cancer 2016; 16: 839 .

[15] Lin HW, Tu YY, Lin SY, Su WJ, Lin WL, Lin WZ, et al. Risk of ovarian cancer in women with pelvic inflammatory disease: a population-based study. Lancet Oncol 2011; 12: 900-904.

[16] Chiou WY, Chen CA, Lee MS, Lin HY, Li CY, Su YC, et al. Pelvic inflammatory disease increases the risk of a second primary malignancy in patients with cervical cancer treated by surgery alone. Medicine (Baltimore) 2016; 95: e5409.

[17] Westrom L, Joesoef R, Reynolds G, Hagdu A, Thompson SE. Pelvic inflammatory disease and fertility. A cohort study of 1,844 women with laparoscopically verified disease and 657 control women with normal laparoscopic results. Sex Transm Dis 1992; 19: 185-192.

[18] Goller JL, De Livera AM, Guy RJ, Low N, Donovan B, Law M, et al. Rates of pelvic inflammatory disease and ectopic pregnancy in Australia, 2009-2014: ecological analysis of hospital data. Sexually Transmitted Infections 2018;

[19] Quintar AA, Mukdsi JH, del Valle Bonaterra M, Aoki A, Maldonado CA, Perez Alzaa J. Increased expression of uteroglobin associated with tubal inflammation and ectopic pregnancy. Fertil Steril 2008; 89: 1613-1617.

[20] Hsu MI, Lin HW. Risk of colorectal cancer in women with pelvic inflammatory disease: a matched cohort study. Bjog 2014; 121: 337-342.

[21] Ewies AA. Risk of colorectal cancer in women with pelvic inflammatory disease: a matched cohort study. Bjog 2014; 121: 1449.

[22] Hsu MI, Lin HW. Authors' reply: Risk of colorectal cancer in women with pelvic inflammatory disease: a matched cohort study. Bjog 2014; 121: 1449-1450.

[23] Risch HA, Howe GR. Pelvic inflammatory disease and the risk of epithelial ovarian cancer. Cancer Epidemiol Biomarkers Prev 1995; 4: 447-451.

[24] Parazzini F, La Vecchia C, Negri E, Moroni S, dal Pino D, Fedele L. Pelvic inflammatory disease and risk of ovarian cancer. Cancer Epidemiol Biomarkers Prev 1996; 5: 667-669.

[25] Cheong KM. Letter by Cheong regarding article, "Association between stroke and patients with pelvic inflammatory disease: a nationwide population-based study in Taiwan". Stroke 2011; 42(e): 560-561.

[26] Grammatikakis I, Evangelinakis N, Salamalekis G, Tziortzioti V, Samaras C, Chrelias $C$, et al. Prevalence of severe pelvic inflammatory disease and endometriotic ovarian cysts: a 7-year retrospective study. Clin Exp Obstet Gynecol 2009; 36: 235-236.

[27] Adriani KS, Brouwer MC, Baas F, Zwinderman AH, van der Ende A, van de Beek D. Genetic variation in the beta2-adrenocepter gene is associated with susceptibility to bacterial meningitis in adults. PLoS One 2012; 7: e37618.

[28] Lim H, Moon A. Inflammatory fibroblasts in cancer. Arch Pharm Res 2016; 39: 1021-1031.

[29] Mierke CT. The fundamental role of mechanical properties in the progression of cancer disease and inflammation. Rep Prog Phys 2014; 77: 076602.

[30] Xie X, Yang M, Ding Y, Chen J. Microbial infection, inflammation and epithelial ovarian cancer. Oncol Lett 2017; 14: 1911-1919.

[31] Shan W, Liu J. Inflammation: a hidden path to breaking the spell of ovarian cancer. Cell Cycle 2009; 8: 3107-3111.

[32] Ingerslev K, Hogdall E, Schnack TH, Skovrider-Ruminski W, Hogdall C, Blaakaer J. The potential role of infectious agents and pelvic inflammatory disease in ovarian carcinogenesis. Infect Agent Cancer 2017; 12: 25.

[33] Skapinyecz J, Smid I, Horvath A, Jeney C, Kardos L, Kovacs P. Pelvic inflammatory disease is a risk factor for cervical cancer. Eur J Gynaecol Oncol 2003; 24: 401-404.

[34] Abdul MA, Shittu SO, Randawa JA, Shehu MS. The cervical smear pattern in patients with chronic pelvic inflammatory disease. Niger J Clin Pract 2009; 12: 289-293.

[35] Iversen L, Sivasubramaniam S, Lee AJ, Fielding S, Hannaford PC. Lifetime cancer risk and combined oral contraceptives: the Royal College of General Practitioners' Oral Contraception Study. Am J Obstet Gynecol. 2017; 216(e): 580-589.

[36] Endometrial cancer and oral contraceptives: an individual participant meta-analysis of 27276 women with endometrial cancer from 36 epidemiological studies. Lancet Oncol 2015; 16: 1061-1070.

[37] Michels KA, Pfeiffer RM, Brinton LA, Trabert B. Modification of the Associations Between Duration of Oral Contraceptive Use and Ovarian, Endometrial, Breast, and Colorectal Cancers. JAMA Oncol 2018; 4: 516-521.

[38] Martino S, Cauley JA, Barrett-Connor E, Powles TJ, Mershon J, Disch D, et al. Continuing outcomes relevant to Evista: breast cancer incidence in postmenopausal osteoporotic women in a randomized trial of raloxifene. J Natl Cancer Inst 2004; 96: 1751-1761.

[39] Lareau SM, Beigi RH. Pelvic inflammatory disease and tubo-ovarian abscess. Infect Dis Clin North Am 2008; 22: 693-708. 